\title{
Synthesis, characterization and cytotoxic activity of S-benzyldithiocarbazate Schiff bases derived from 5-fluoroisatin, 5-chloroisatin, 5-bromoisatin and their crystal structures
}

\begin{abstract}
Schiff bases were prepared from S-benzyldithiocarbazate with 5-fluro-, 5-chloro- and 5bromoisatin. All are potential tridentate nitrogen, oxygen, sulfur donors. They were found to be selectively active against MCF-7 cell line (Human non-metastatic mammary gland adenocarcinoma cell line). The bromide and fluoride compounds were the most active with IC50 values of $6.40 \varepsilon \mathrm{M}(2.6 \mathrm{\varepsilon g} / \mathrm{mL})$ and $9.26 \varepsilon \mathrm{cM}(3.2 \mathrm{\varepsilon g} / \mathrm{mL})$ respectively while the chloride derivative was weakly active with an IC50 value of $38.69 \varepsilon \mathrm{M}(14.0 \mathrm{\varepsilon g} / \mathrm{mL})$. The cytotoxic activity of the halo substituted isatins against the breast cancer cell lines tested is in the order of $\mathrm{Br}>\mathrm{F}>\mathrm{Cl}$. Planarity of the isatin ring in the Schiff bases can be arranged in the following order SB5FISA > SB5CIISA > SB5BrISA while the perpendicularity of the benzyl ring towards the dithiocarbazate plane can be ordered as follows, SB5FISA > SB5BrISA > SB5CIISA.
\end{abstract}

Keyword: Dithiocarbazate; Schiff base; Isatin; MCF-7; Structure 